\title{
Effect of weight loss on mitochondrial defects in the ageing human colon
}

\author{
S.P. Breininger, L. Greaves, D. Turnbull and J.C. Mathers \\ Newcastle University, The Medical School, Framlington Place, NE2 4HH, United Kingdom
}

Colorectal cancer (CRC) is the $3^{\text {rd }}$ most common cancer worldwide ${ }^{(1)}$. CRC is initiated in colonocytes and, with advancing age, colonocytes accumulate mitochondrial mutations which contribute to age-related dysfunction and to increased cancer risk ${ }^{(2)}$. Obesity, and its lifestyle determinants, physical inactivity and poor diet, increase CRC risk ${ }^{(3)}$. The effects of weight loss by bariatric surgery on mitochondrial defects in human colonoctyes are unknown.

Mitochondrial defects in the colorectal mucosa are i) elevated in obese individuals compared with non-obese age-matched individuals and ii) reduced following weight loss by bariatric surgery.

Obese patients listed for bariatric surgery and age-matched healthy non-obese individuals (Controls) were recruited at North Tyneside General Hospital. Rectal mucosal biopsies were collected at baseline and six months post-surgery from obese participants and at baseline only from Controls. Mitochondrial oxidative phosphorylation proteins complex 1 and 4 and mitochondrial mass were quantified by immunofluorescence after staining with validated antibodies.

Data were available for 16 Controls and for 31 obese participants pre- and post-bariatric surgery, all aged 21-65 years. Mean BMI of the pre-surgery obese group was significantly higher than for Controls (means 41.9 and 25.9, respectively) $(\mathrm{p}=0.001)$ and bariatric surgery resulted in mean $26 \mathrm{~kg}$ weight loss. The prevalence of complex $4(\mathrm{p}=0.039)$ and mitochondrial mass $(p=0 \cdot 010)$ deficiency was significantly greater in older $(>48 \mathrm{y})$ compared with younger $(\leqslant 48 \mathrm{y})$ individuals. Obese individuals had significantly more complex 1 depleted crypts $(9.2 \%)$ compared with Controls $(0 \%)(\mathrm{p}=0.046)$. In addition, the obese had significantly fewer crypts with normal complex $4(96.9 \%)$ and mitochondrial mass $(93.8 \%)$, and more crypts with complex $4(3.1 \%)$ and mitochondrial mass $(6.2 \%)$ deficiency compared with Controls $(100 \%, 99.9 \%, 0 \%$ and $0.04 \%$ respectively) $(\mathrm{p}=0.03)$. However, weight loss did not change significantly the concentrations of oxidative phosphorylation proteins.

Advancing age and greater adiposity lead to significantly more complex 1 and 4 deficiencies in colonocytes but, at least after 6 months, weight loss by bariatric surgery had no significant effect.

S.P.B. acknowledges PhD studentship funding by Medical Research Council.

1. Ferlay J, Soerjomataram I, Dikshit R, Eser S, Mathers C, Rebelo M, et al. (2015) Cancer incidence and mortality worldwide: sources, methods and major patterns in GLOBOCAN 2012. Int $J$ Cancer 136(5), E359-86.

2. Greaves LC, Nooteboom M, Elson JL, Tuppen HA, Taylor GA, Commane DM, et al. (2014) Clonal expansion of early to mid-life mitochondrial DNA point mutations drives mitochondrial dysfunction during human ageing. PLoS genetics 10(9), e1004620.

3. World Cancer Research Fund / American Institute for Cancer Research. Food, Nutrition, Physical Activity, and the Prevention of Cancer: a Global Perspective. Washington DC, 2007. 